\title{
Vehicle Feature Extraction and Vehicle Classification Recognition
}

\author{
Wang Yi, Zhu Rui \\ Hubei Three Gorges Polytechnic, Yichang, Hubei, 443000
}

Keywords: vehicle location; windows location; vehicle feature extraction; vehicle classification and recognition

\begin{abstract}
The localization of vehicle is mainly the location of vehicle and the accurate localization of eigenvalue. Both methods are generated by the horizontal and vertical projection of the image. The projection region is a rectangle. The vehicle location is combined with the feature extraction process, and the key is to find the first location, which is more important to determine the segmentation threshold localization process itself. The threshold can be adjusted according to the distance between the camera and the vehicle.
\end{abstract}

\section{Windows Location Algorithm}

We still adopt the vehicle windows location algorithm based on the color extraction method. Color information extraction device is to separate the color region of the image edge and the color block with uniform chromaticity from the image. Color information is not only the recognition result that we need, but also can help us locating the vehicle, so if we want the location to be accurate, we need to design a color extraction algorithm. In practice, you should first choose a color model, the most widely used is the RGB model. We need to extract the color information of the windows. The chromaticity of windows and shadows are very close to the gray line, which means that their color value R, G,B is similar, that is to say, the windows and shadows have similar color characteristics. However, their geometric properties are very different, so they can still be distinguished. The key of windows location depends on the comprehensive use of gray information and weak reflected light.

In the first step of the algorithm, the rectangle in the specified range is abstract, which is the region to be recognized in the established mathematical model. In order to eliminate the influence of contingency on the recognition result, we can identify in column order and find more rows or columns. At first search for $\mathrm{N}$ rows (columns), if there are more than $\mathrm{M}$ row (column) values meet the search requirements, and then it is considered to have found the required split point. The values of $\mathrm{N}$ and $\mathrm{M}$ are selected according to the experimental experience, and the quality and accuracy of the localization results are directly affected by the selection of the stable algorithm. The reason we want to position ourselves is The more characteristic values can be located in distinguishing and accurately locating the feature values, the more advantageous the model classification method can be found.

The windows location method, as we said earlier, using the pattern recognition of the profile curve of the vehicle to identify the vehicle model. However, in the driveway, the contour of the vehicle is difficult to be accurate because of the surrounding pedestrians, the landscape of the road and the mutual occlusion between the vehicles. We use the top view of the car to identify vehicle models from the identified position and size of the windows, and use the different HSV chromaticity to locate the windows, and propose a chromatic aberration-based windows positioning method.

The characteristics of the image are separable, the different features of the image can be obtained by different angles, some are the natural features of the image itself, such as the gray value of the pixel itself, all of which are immutable. In addition, some of the eigenvalues are changed, so we have to choose the same quantity as much as it is more stable. It is necessary to separate the car windows out of the car image. We analyze the features of the windows area. We can draw the following conclusions: there are some vehicles, such as light color vehicles, their windows color is 
very different from the paint color of the car. For example, a white car, getting off in the sun. The color of windows glass is usually dark (usually gray and black). We choose the front window to analyze, its shape is similar to the rectangle, and the width of the window is about the same as the car body width. We can roughly think that finding the position of the front window is to find a rectangular area with different chroma on the top of the car. And because the front window is always in the front of the car in a complete car image, we can narrow the search range.

According to the method of looking for the front window of the car, it is possible to locate it automatically. But because of the existence of a wide variety of vehicles, we still encounter some problems in implementation: for example, the color of the vehicle is various, the color of the dark black vehicle is very similar to the color of the window. It is difficult to distinguish between the two in a situation like this. There are also some vehicles with roof windows, making it difficult to define the scope of the front window.

According to the characteristics of the vehicle windows mentioned above, we can find out the pixel points with the same value of RGB in the rectangular range of the vehicle. It is considered that the region composed of such stable gray points is the windows area. But in the actual programming recognition we find that this can cause a lot of errors because there may be some examples where the pixel area is similar to the gray value of the windows area. Based on the above situation, we can only limit the range of RGB values in the windows area in order to obtain correct recognition results. But there are some cases where the reflective characteristics of the body part of a dark vehicle are very similar to those of the windows part, and in this case, we have some limitations to use. Let's look at the window location results of the two vehicles in figure 1:

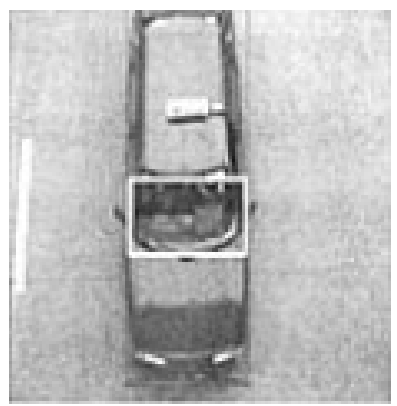

a)

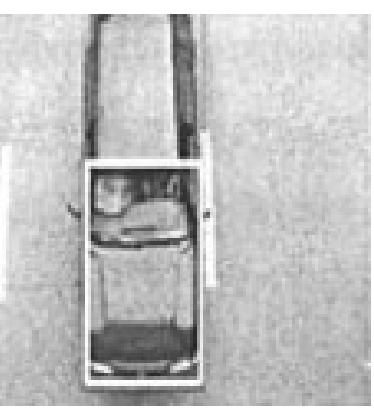

b)

Figure 1 Windows positioning based on reflective characteristics

It can be found from figure 1 that the result of locating the vehicle windows is correct in the view a) and that in the view b) the recognition result of the vehicle is wrong. We find that the reflection characteristics of the windows glass and the front part of the vehicles are very similar by analyzing the error cause of the view b), which makes the program mistake that both of them are the same windows area. This is due to the fact that in the view a), the original light color vehicles in the diagram are quite different from the reflectivity of the windows, while that in the view b),there is a dark vehicle, and the difference in the reflectivity between the two is not significant.

It is for these reasons that it is understood that this approach is not universal because:

1) not anti-interference, dark vehicles body and windows materials are different, but they have similar reflectivity of light;

2) the range of application is not wide, which is more suitable for the identification of light painted vehicles such as white, yellow and pink, but the recognition rate for dark vehicles (such as black and dark blue) is very low.

Although this method is not universal, and at least it has a higher recognition rate for light colored vehicles. We can consider improving it so that it can be applied to all vehicles in a certain range. We can use a threshold method to achieve it. It is divided into two steps:

1) According to the specific environmental needs, determine the correct threshold.

2) Comparing the threshold to the value of the pixel to find out the range of the region to be segmented.

Finding the appropriate threshold is the key, and the selection of the threshold is the key to 
identify the correct or not. After the threshold is selected, the process of comparing each pixel value with the threshold value can be synchronized, and the final binary black and white image can be identified and displayed.

On the basis of the above design, we set the threshold point of 30 as the boundary. When $\mathrm{P}>30$, it is a light-colored vehicle, and the windows location algorithm with reflective characteristics is used directly to identify it; when $\mathrm{P} \leq 30$, it is a dark vehicle. Automatic threshold method is used to complete localization. While this approach is good, there are still some omissions, as can be clearly seen from figure 2:
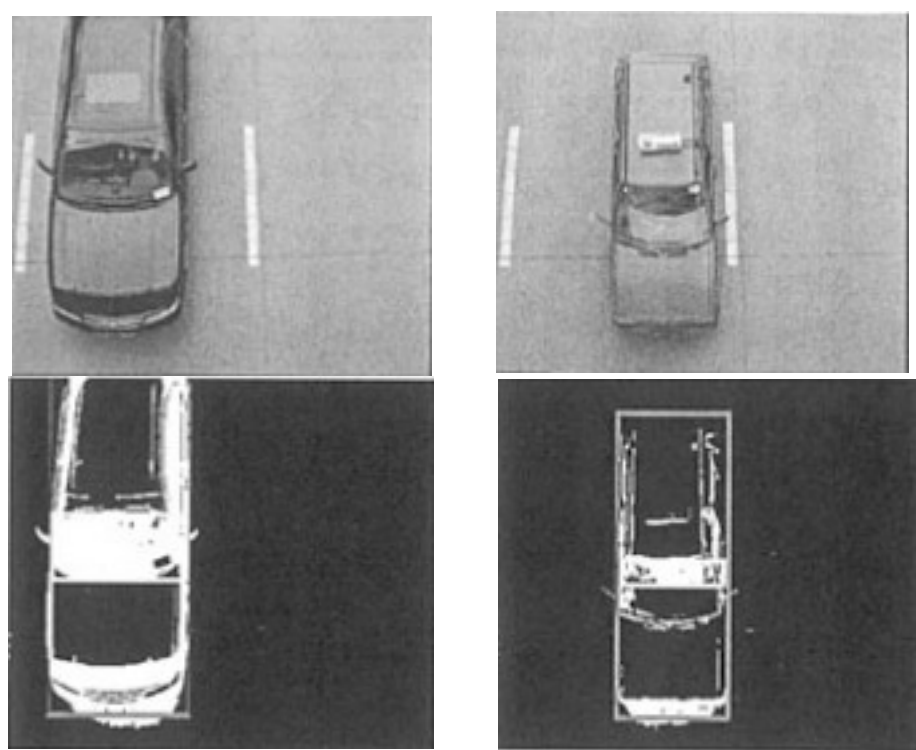

Figure 2 Automatic threshold positioning windows

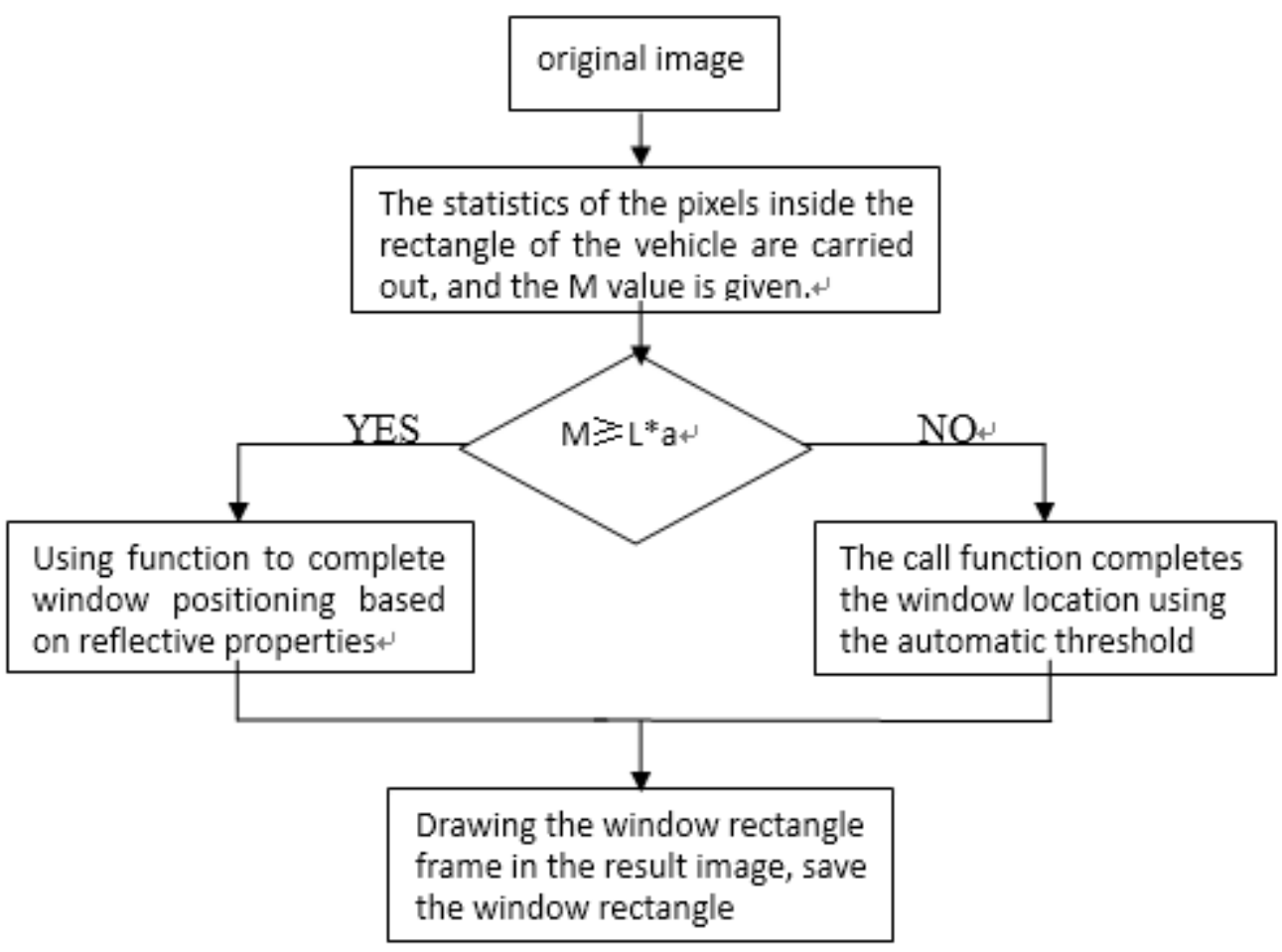

Figure 3 Windows location flow chart

In the above two images, the average gray level in the vehicle rectangle is 20. According to the flow chart, they all use the same method-automatic threshold method to locate. Well, the difference in recognition results is due to the limitations of the automatic threshold method itself. At the time of the design, we think that the gray value is in the range of $18 \sim 20$ for dark vehicles, and we only use this method as an empirical verification now. At present, the correctness of this method can not 
be fully verified. The main reason is that it is biased only by the gray value, for example, the judgment of black vehicle, because of the high reflectivity of black paint, the car body paint reflects sunlight, will make it. The color appears a little lighter, gray value and part of the light-colored vehicle color is very close. This will result in inaccurate test results. In order to make up for the deficiency of the initial design, we need a more accurate method to identify the color of the car. The final design flow chart is as figure 3, The recognition results are shown in figure 4:
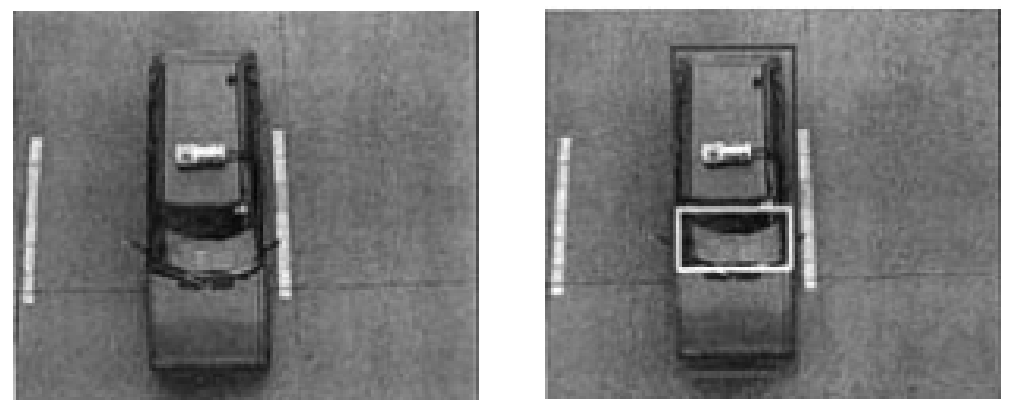

Figure 4 Windows location results

\section{Vehicle Feature Extraction}

In vehicle recognition, it is very important to select the appropriate eigenvalue, which will slow down the processing speed and increase the computation of the algorithm, thus reducing the recognition rate.

There are two methods for extracting eigenvalues: first, according to certain principles, the average gray value obtained in different positions is used as a feature. Two, the physical property is converted to a text or numerical form in the case of full understanding of the image features, because such a form is easier to identify.

For the feature extraction of vehicle, a better method is to collect a large number of data samples in order to extract the vehicle features accurately from a technical point of view. But from the user's point of view, the rapid identification of models means that the data sample can not be too much. At the same time, we will bring in some redundant information when we collect data parameters, in order to achieve the purpose of fast and accurate recognition. The redundancy of information must be very small, and the selection of characteristic value also needs to be universal. Based on such requirements, you should be aware of the following three conditions when selecting eigenvalues:

1) distinctiveness, which means that different types of vehicles need to extract different kinds of eigenvalues;

2) similarity, which means that the selected eigenvalues of the same vehicle should be as consistent as possible;

3) simplicity, too much selection of eigenvalues will bring burden to vehicle recognition, and the selection of eigenvalues should be purposeful.

The above three conditions are summarized: distinctiveness is the basis, similarity is the guarantee, and simplicity is the key.

There are more eigenvalues in vehicles, such as the length, width, high information and displacement of the vehicle can be selected. But from the point of view of easy to operate, the information of vehicle displacement is not the key to determine the size of the vehicle, and it is more difficult to obtain its value. So we choose the simple eigenvalue, that is, length or width, as the criterion. Why choose such a value, because it is easy to get through the camera image. We can be divided into different types of vehicles according to different length ratios.

\section{Vehicle Classification and Recognition}

After a lot of operations, the vehicle model is classified and recognized. The feature value is extracted and the template matching method is established to identify the vehicle model.

The design will be divided into three types of models, small cars, medium-sized cars, large cars. 
The size of the three models is obviously different, and generally, different models have the following characteristics:

Table 1 Vehicle profile dimensions

\begin{tabular}{|c|c|c|c|}
\hline \multirow{2}{*}{ Vehicle type } & \multicolumn{3}{|c|}{ Vehicle profile size (Unit m) } \\
\cline { 2 - 4 } & Length & Width & Altitude \\
\hline Small Vehicle & 3.20 & 1.60 & 1.80 \\
\hline $\begin{array}{c}\text { Medium } \\
\text { Vehicle }\end{array}$ & 5.00 & 2.00 & 2.20 \\
\hline Large Vehicle & 8.70 & 2.50 & 4.00 \\
\hline
\end{tabular}

Here, we shoot the roof, according to the previous windows location method, according to the vehicle front window position in the whole vehicle to determine the model, referring to the above table summarized vehicle size to locate: Windows position (windows coordinates-front coordinates / total vehicle length) In the test, the car length is chosen as the judgment condition, and when the length is not easy to obtain, the car width can be used as the judgment condition.

In order to achieve a higher recognition rate, we use the method of background subtraction to locate the vehicle. The resolution of the recognizable image is $800 * 600$. The RGB value of the vehicle model is compared with the RGB value of the background image, and whether there is a big color difference between them is analyzed. When the color difference is less than the set threshold, the pixel can be considered as the vehicle target and its RGB value is 255. Otherwise, the pixel is assigned a background RGB value of 0 . The image is subjected to grayscale, binarization, dilation, opening and closing operations, and the precise roof length and floor length can be obtained. The ratio of the length to the bottom of the vehicle is taken as the judging condition of the vehicle model, and a small car when the ratio is $0.2 \leq$ percent $\leq 0.5$; when $0.5<$ percent $\leq 0.8$ the medium type car is determined; when percent $>0.8$, this is a large car.

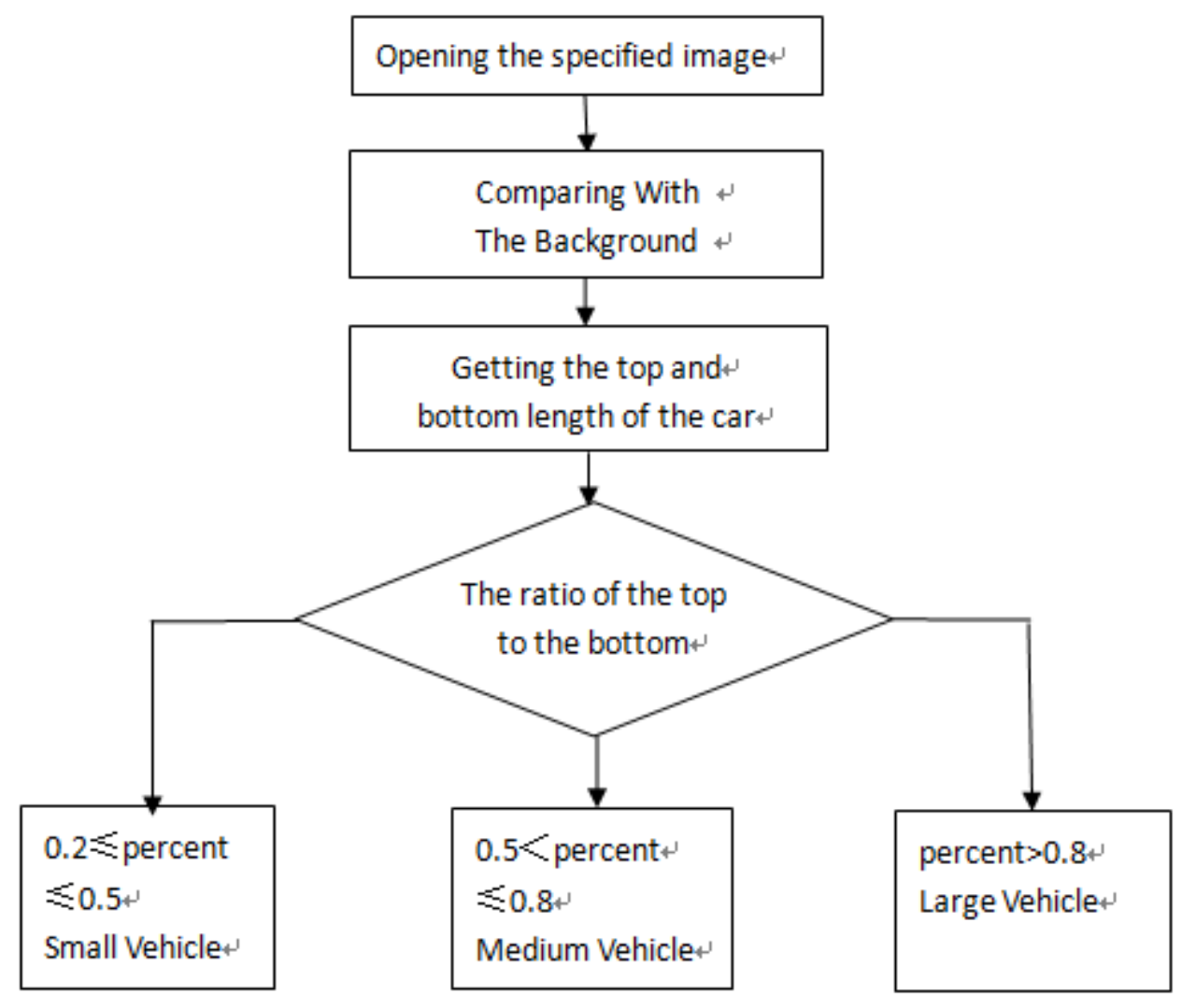

Figure 5 Algorithm flow chart 


\section{Acknowledgments}

Fund Project: Yichang City Applied Basic Research Project, "Research on vehicle license Plate pattern recognition in Expressway ETC system", project number: A14-302-b02

\section{References}

[1] Zhou Zhengjie, Wang Runsheng. Feature extraction and recognition method based on contour shape. Computer Engineering and Application.

[2] Chen Aibin. Recognition of vehicle types based on characteristic cars, 2004 (5)

[3] Shen Yongwu, automatic vehicle classification system based on image recognition. Zhejiang University, 2008

[4] Zhou Zhengjie, Wang Runsheng. Feature extraction and recognition method based on contour shape. Computer Engineering and Application. 